\title{
Are emotional action tendencies and attentional bias related to temperament dimensions in patients with borderline personality disorder?
}

\author{
Ellen Sleuwaegen ${ }^{\mathrm{a}, \mathrm{b}, *}$, Wouter Hulstijn ${ }^{\mathrm{b}}$, Laurence Claes ${ }^{\mathrm{b}, \mathrm{c}}$, Marlies Houben ${ }^{\mathrm{c}}$, Amarendra Gandhi ${ }^{\mathrm{c}}$, \\ Ann Berens ${ }^{\mathrm{a}}$, Bernard Sabbe ${ }^{\mathrm{a}, \mathrm{b}}$

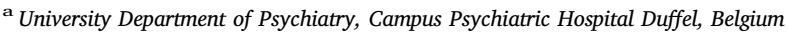 \\ ${ }^{\mathrm{b}}$ Faculty of Medicine and Health Sciences, University of Antwerp, Antwerp, Belgium \\ ${ }^{\mathrm{c}}$ Faculty of Psychology and Educational Sciences, KU Leuven, Leuven, Belgium
}

\section{A R T I C L E I N F O}

\section{Keywords:}

Temperament dimensions

BPD subtypes

Emotional action tendencies

Attentional bias

Emotional Stroop Task

Approach-Avoidance Task

\begin{abstract}
A B S T R A C T
Disturbances in emotion regulation have been identified as a core feature of patients with a borderline personality disorder (BPD). Findings of studies using experimental measures of emotion processing are mixed, which may be partially explained by the heterogeneity of the BPD population. To address this issue, we investigated differences in experimental measures of emotional action tendencies (approach-avoidance behaviour) and attentional bias to emotional stimuli in BPD subtypes. Data of the Approach-Avoidance Task (AAT) and the Emotional Stroop Task (EST) were collected in 140 BPD patients, previously clustered into four BPD subtypes based on temperament dimensions. We investigated (1) the relationship between temperament dimensions and the performance on the AAT and EST and (2) compared performance on these tasks in previous defined BPD subtypes. The results of the present study demonstrated a positive relationship between effortful control (EC) and AAT effect-scores. A higher level of EC was positively associated with a general emotional action tendency towards faces with directed gaze, even when controlling for gender, age and BPD severity. Preliminary results on the comparison of the BPD subtypes demonstrated no significant differences in AAT and EST performance. These findings emphasize the relevance of EC in emotional action tendencies in BPD patients.
\end{abstract}

\section{Introduction}

Emotional dysregulation is viewed as a core feature of borderline personality disorder (BPD; e.g., Glenn and Klonsky, 2009). Emotion regulation deficits have been linked to impulsive or maladaptive behaviours such as non-suicidal self-injury, suicidal behaviour and treatment drop-out (e.g., Schmahl et al., 2014). Although characteristics of emotion processing in BPD have been extensively investigated, results of studies using experimental measures are mixed (Rosenthal et al., 2008). The inconclusive results may be partially explained by the heterogeneous traits present in the BPD population.

In an earlier study (Sleuwaegen et al., 2017) an attempt was made to disentangle the heterogeneity in BPD based on differences in patients temperament. Reactive and regulative temperament traits were measured using the Behavioral Inhibition and Behavioral Activation Scales (BIS/BAS; Carver and White, 1994) and the Effortful Control Scale (ECS-ATQ; Evans and Rothbart, 2007) respectively. Results of the study enabled four BPD subtypes to be distinguished. The 'Low Anxiety' subtype $(21 \%)$ was characterized by low levels of BIS reactivity (low punishment sensitivity/low avoidance). Patients of this subtype reported less anxiety, more expression of emotions and had higher scores relating to antisocial personality disorder (PD) features. The 'Inhibited' subtype (24\%) was characterized by low levels of BAS reactivity (low reward sensitivity/low approach). These patients reported less hostility, less expression of emotions, and had higher scores relating to avoidant PD features. The last two subtypes showed similar reactive temperaments with moderate levels of BIS and BAS, but differed in their regulative temperament. The 'High Self-control' subtype (10\%) was characterized by very high levels of effortful control. Patients of this subtype reported fewer clinical symptoms, more adaptive coping strategies and fewer comorbid personality disorder features. On the other hand, the 'Emotional/Disinhibited' subtype (45\%) demonstrated very low levels of effortful control. These patient were characterized by higher levels of anxiety, less adaptive coping strategies and higher cluster B (histrionic) PD features (see Sleuwaegen et al., 2017). Thus, the two subgroups presenting with the lowest and highest levels of psychopathology ('High Self-control' and 'Emotional/Disinhibited') only differed on the level of effortful control.

\footnotetext{
* Corresponding author.

E-mail address: Ellen.sleuwaegen@uantwerpen.be (E. Sleuwaegen).
} 
In support of this, evidence from an earlier study found that levels of effortful control contributed to BPD symptoms (Hoermann et al., 2005). Using self-reported measures, BPD patients with the highest level of effortful control reported the least symptoms and fewest problems in interpersonal functioning and personality organization, whereas those with the lowest level of effortful control reported the most problems in these areas. An additional study demonstrated that lower effortful control and higher BIS in BPD patients resulted in worse performance on a task measuring cognitive control (conflict resolution) (Posner et al., 2002). Preti et al. (2016) therefore concluded that failure of regulatory processes negatively affects performances.

As evidence relating to emotion processing in BPD is inconclusive, temperament dimensions, specifically effortful control might account for some important differences in presentation and reactions to emotional stimuli as measured by performance on experimental tasks (Posner et al., 2003).

Recent studies in BPD patients have demonstrated a biased processing of emotional stimuli (Winter, 2016). Attentional bias to emotional stimuli is often investigated with interfering emotional stimuli being presented during a task. For example, in the Emotional Stroop Task (EST), patients have to name the colour of ink in which emotional or neutral words are printed as fast as possible. In this task, the emotional content of the words may capture attention. Kaiser et al. (2017) found that patients with BPD required more time to name the colour of negative words during the EST, suggesting a negative attentional bias in BPD patients. In addition, Portella et al. (2011) reported that higher severity of BPD psychopathology led to more difficulties in processing information measured by the EST.

Another important emotion-related process is the response to emotional stimuli, often labelled emotional action tendencies. Research on this topic has grown widely since the introduction of the ApproachAvoidance Task (AAT; Rotteveel and Phaf, 2004), where participants have to respond to pictures of happy or angry faces by pushing or pulling a joystick depending on the emotional expression. In general, people respond faster if asked to approach a happy face and to avoid an angry face, compared with the task of approaching an angry face and avoiding a happy face, which need more control to apply counterintuitive action (Roelofs et al., 2009). Disturbed approach-avoidance tendencies on the AAT are seen in different patient samples. In socially anxious patients, for example, increased avoidance tendencies toward angry and happy faces are observed, whereas in depressed patients a decreased overall approach tendency is observed (Radke et al., 2014; Roelofs et al., 2010). As far as we know, no research to date has explored the emotional action tendencies with the AAT in BPD patients.

As indicated by previous studies, the distinction between BPD patients based on temperament dimensions may provide a framework to better understand the mixed findings relating to emotion processing in BPD (Suvak et al., 2012; Unoka and Richman, 2016, Winter, 2016). To test this, (1) the relationship between temperament dimensions and performance on EST and AAT was investigated and (2) a comparison was made between the previously mentioned BPD subtypes based on temperament dimensions on these two experimental tasks.

Concerning research question 1 , the associations between AAT and EST-effects and temperament dimensions were investigated. Since gender, age, and BPD severity may influence the aforementioned associations (e.g., Price et al., 2012), it was explored whether the associations would remain after controlling for these variables. The first hypothesis of this research question was that there would be positive associations between effortful control and both experimental measures (Posner et al., 2003) above and beyond gender, age and BPD severity. It was additionally hypothesized that the level of effortful control would be related to AAT performance, since this task demands some voluntary counterintuitive actions. However, this association was mainly expected in the condition in which faces with a direct gaze were used, since they evoke more intense automatic activation (Roelofs et al., $2009 ; 2010)$. The final hypothesis of this research question was that EST performance may be explained by the level of effortful control, since this task depends on the ability to make subdominant responses.

Concerning research question 2, the four BPD subtypes were compared on their AAT and EST performance. Although the current study compared subtypes with limited sample sizes, it can provide preliminary evidence for differences on action tendencies and attentional bias to emotional stimuli between BPD subtypes. Since this part of the study was more explorative in nature, the only hypothesis was that there would be differences between the two BPD subtypes with contrasting effortful control. The High Self-control subtype (high EC) would perform better on the AAT and EST, while the Emotional/ Disinhibited subtype (low EC) would display more approach-avoidance deficits and attentional bias to emotional stimuli (Portella et al., 2011; Posner et al., 2003).

\section{Methods}

\subsection{Participants and procedure}

The present study is an extension of research on BPD subtypes based on reactive and regulative temperament (see Sleuwaegen et al., 2017). The original sample consisted of 146 patients ( $85.6 \%$ female), recruited from two psychiatric hospitals in Belgium and diagnosed with BPD by means of the Structured Clinical Interview for DSM-IV Axis II Personality Disorders (SCID-II; First et al., 1997). Within the context of the present study, the AAT and EST were administered in a session following the interview-session. Due to technical error, two patients (of the 'Emotional/Disinhibited' subtype) were excluded, resulting in a dataset of 144 patients. Of this BPD sample, 125 (86.8\%) are female and 19 are male (13.2\%), with a mean age of 29.30 years $(S D=8.35$, range 18 to 65 years). 'Low Anxiety' $(n=31)$, 'Inhibited' $(n=34)$, 'High Selfcontrol' $(n=15)$ and 'Emotional/Disinhibited' subtypes $(n=66)$ did not significantly differ with regard to gender, education, marital status or type of medication used (see Sleuwaegen et al., 2017). The study was developed in accordance with the Declaration of Helsinki and approved by the Ethics Committee of Antwerp University and the local ethical committee of the participating hospitals.

\subsection{Measures}

Reactive temperament was assessed by means of the Behavioral Inhibition/Behavioral Activation System Scales (BIS/BAS; Carver and White, 1994; translated into Dutch by Franken et al. (2005)). The BIS/ BAS scales consist of 24-items, rated on a 4-point Likert scale, of which seven items assess BIS reactivity (sensitivity to punishment) and 13 items assess BAS reactivity (sensitivity to reward). The BIS and BAS scales demonstrated acceptable internal consistency in the present sample (both $\alpha=0.75$ ).

Regulative temperament was assessed by means of the Effortful Control Scale (ECS) from the short form of the Adult Temperament Questionnaire (ATQ; Evans and Rothbart, 2007), consisting of 19-items, rated on a seven-point Likert scale. The alpha coefficient of the ECS in the present study was 0.78 .

Severity of symptoms related to the Borderline Personality disorders were assessed by means of the Assessment of DSM-IV Personality Disorders (ADP-IV; Schotte and De Doncker, 1994), a 94-item Dutch self-report questionnaire used to assess the presence of 10 personality disorders defined in the DSM-IV-TR. Dimensional scores were computed by summing the trait scores on the individual items for each PD scale. The alpha coefficients in the present study for the BPD was $\alpha=0.67$.

Action tendencies to emotional stimuli were assessed by means of the gaze variant of Approach-Avoidance Task (AAT; developed by Heuer et al. (2007); Radke et al. (2013)). In this task, patients have to respond to pictures of facial expressions (happy, angry) of eight different actors ( 4 male, 4 female), with different gazes (direct or averted gaze), presented on a computer screen. Patients were instructed to 
respond to the emotional expression of the face by pushing a joystick away from themselves as fast as possible (avoid) which consequently shrinks the picture; or pulling a joystick toward themselves (approach) and enlarging the picture. All patients performed two blocks of trials with opposite instructions. In the affect-congruent block, patients had to pull the joystick (approach) when presented with a happy face and to push the joystick (avoid) when presented with an angry face. The affectincongruent block required the opposite actions, namely to pull following an angry faces and push following a happy faces. Each block consisted of 20 practice trials and 96 test trials. The order of the blocks was counterbalanced across patients. Reaction times (RTs) were transformed into their natural logarithm. Median RTs were calculated for movements (push, pull) for different emotions (happy, angry) and different gazes (direct, averted). The AAT effect scores were calculated by subtracting participants' median RTs in the pull conditions from median RTs in the push conditions. A positive effect score reflects facilitated approach patterns whereas negative effect scores represent faster avoidance patterns. To investigate a general emotional action tendency, AAT effect-score of combined emotions (push-pull happy minus pushpull angry) were calculated (for an extensive review of this task, see meta-analysis by Phaf et al., 2014).

Attentional bias was assessed by means of the Emotional Stroop Task (EST; e.g., Williams et al., 1996). The standard card version of the EST was used as opposed to the computerised version, since verbal responses seem to show higher interference scores than button responses (Kaiser et al., 2017). Three A4 cards were composed, each containing another emotional valence. The order in which the three cards were presented was counterbalanced across patients. Each card consisted of $10 \times 10$ neutral, negative, or positive words printed in red, yellow, green or blue ink. The patients were asked to name the colour of the ink in which the words were printed as fast as possible. The total time needed to name the colours of a card was recorded. The resulting three mean colour naming times for neutral, positive or negative words are the sum of the reaction time and the duration of the verbal output. The total time was divided by the number of words $(=100)$, representing a colour naming per word in milliseconds. Positive attentional bias was defined as the difference between positive colour naming time and neutral colour naming time; negative attentional bias as the difference between negative and neutral colour naming time. Higher scores indicate more attentional bias to emotional stimuli (for an extensive review of this task see meta-analysis by Kaiser et al. (2017)).

\subsection{Statistical analyses}

All analyses were performed using SPSS (v24). AAT trials with an extreme RT $(<150 \mathrm{~ms}$ or $>1500 \mathrm{~ms}$ or exceeding $2.5 \mathrm{SD}$ above the individual mean) or an extreme MT ( $>400 \mathrm{~ms}$ ) were excluded $(<4 \%)$. Four participants (of the Emotional/Disinhibited BPD subtype) with extreme RTs were excluded. RTs for the AAT and EST were transformed into their natural logarithm (all reported RTs are $\ln (\mathrm{RT}) \mathrm{s}$ ). Statistical analyses were performed on median RTs (Roelofs et al., 2010).
To answer the first research question, i.e. the associations between the temperament dimensions (BIS, BAS and EC) and the experimental tasks (AAT and EST effects), Spearman's correlations were calculated.

In addition, three separate hierarchical linear regression analyses were performed with (1) AAT effect-scores (push-pull RT differences) of happy faces with direct gaze, (2) AAT effect-scores of angry faces with direct gaze and (3) general AAT effect-scores (push-pull RT happy minus push-pull RT angry) as dependent variables and temperament dimensions (BIS, BAS and EC) were simultaneously entered in block 1 as independent variable and BIS, BAS, EC, gender, age and BPD severity entered in block 2 as independent variable.

Next, two separate hierarchical linear regression analyses were performed for EST effects, (1) with positive bias (positive minus neutral) and (2) with negative bias (negative minus neutral) as dependent variables and temperament dimensions (BIS, BAS and EC) simultaneously entered in block 1 as independent variable and BIS, BAS, EC, gender, age and BPD severity entered in block 2 as independent variables.

To answer the second research questions, i.e. to compare the BPD subtypes with respect to the AAT/EST, three separate hierarchical linear regression analyses were performed (1) with general AAT effectscores (push-pull RT happy minus push-pull RT angry), (2) with positive bias (positive minus neutral) and (3) with EST negative bias (negative minus neutral) as dependent variables and BPD subtypes (binary coded and the Emotional/Disinhibited subtype as reference BPD subtype) simultaneously entered in block 1 as independent variable and BIS, BAS, EC, gender, age and BPD severity entered in block 2 as independent variable.

\section{Results}

\subsection{Preliminary analyses of the AAT and EST effects}

AAT effect-scores were analyzed with a repeated-measure ANOVA with emotion (happy, angry), gaze (direct, averted). Results showed a significant main effect of emotion $[F(1,139)=9.27, p=0.003]$, with the expected pattern of approach of happy faces $(M=33.53)$ and avoidance of angry faces $(M=-9.81)$. Increased AAT effects for faces with direct gaze were reflected by a significant main effect of gaze $[F$ $(1,139)=5.99, p=0.016]$. There was a significant interaction between emotion and gaze $[F(1,139)=3.91, p=0.049]$, caused by happy faces with direct gaze. Since these findings pointed to a greater effect of faces with direct gaze, it allowed us to focus on the direct gaze condition and omit the averted gaze condition in further analyses.

EST response time scores were analyzed with a repeated-measure ANOVA and revealed a significant difference between EST response time for neutral, positive and negative words $[F(2,137)=12.44$, $p<0.001]$, with longer response time for negative words $(M=807 \mathrm{~ms})$ and positive words $(M=789 \mathrm{~ms})$ compared to neutral words $(M=753 \mathrm{~ms})$. This result provides evidence for the measurement of attentional bias for emotional words with this EST.

Table 1

Correlations between temperamental dimensions and AAT and EST effects.

\begin{tabular}{|c|c|c|c|c|c|c|c|c|}
\hline & BIS & BAS & EC & AAT-effect Happy_Direct & AAT-effect Angry_Direct & AAT-effect H_A_Direct & EST Pos-Neutr & EST Neg-Neutr \\
\hline BIS & 1 & .062 & -0.027 & -0.083 & -0.046 & -0.027 & .070 & .072 \\
\hline BAS & .062 & 1 & $-0.386^{* *}$ & -0.059 & .136 & -0.160 & -0.022 & -0.031 \\
\hline EC & -0.027 & $-0.386^{* *}$ & 1 & $.231^{* *}$ & $-0.221 * *$ & $.283^{* *}$ & -0.097 & -0.070 \\
\hline AAT-effect Happy_Direct & -0.083 & -0.059 & $.231 * *$ & 1 & $-0.420 * *$ & $.850 * *$ & .007 & .126 \\
\hline AAT-effect Angry_Direct & -0.046 & .136 & $-0.221 * *$ & -0.420 & 1 & -0.807 & .086 & -0.057 \\
\hline AAT-effect H_A_Direct & -0.027 & -0.160 & $.283^{* *}$ & $.850^{* *}$ & -0.807 & 1 & -0.059 & .111 \\
\hline EST Pos-Neutr & .070 & -0.022 & -0.097 & .007 & .086 & -0.059 & 1 & $.316^{* *}$ \\
\hline EST Neg-neutr & .072 & -0.031 & -0.070 & .126 & -0.057 & -0.111 & $.316^{* *}$ & 1 \\
\hline
\end{tabular}

Note. ${ }^{* *} p<0.01$ 


\subsection{Associations between temperament dimensions and AAT, EST effects}

Table 1 presents the Spearman correlations between the self-reported temperament dimensions (BIS, BAS and EC) and AAT effectscores on emotional faces with direct gaze and EST attentional bias scores in the total BPD sample. The results of the correlations showed that effortful control was negatively related to BIS and positively related to the AAT effect-scores towards happy and angry direct faces and to the general AAT effects scores.

To investigate which temperament dimension had the strongest association with the AAT effect-scores (with direct gaze), three hierarchical linear regression analyses were performed.

The first hierarchical regression analysis, with AAT effect-scores towards happy faces as dependent variable and BIS, BAS and EC entered in block A, revealed a statistically significant model $[F(3,136)=2.91$, $\left.p=0.037, R^{2}=0.040\right]$. The level of effortful control made a significant contribution to the model [ $\beta=0.225, t=2.48, p=0.014]$, BIS and BAS not (both $p>0.269$ ). However, after entry of gender, age and BPD in the second block, the model was not significant anymore $[F$ $\left.(3,136)=1.84, p=0.096, R^{2}=0.035\right]$.

The second hierarchical regression analysis, with AAT effect-scores towards angry faces as dependent variable and BIS, BAS and EC entered in block 1 , did not provide a significant model $[F(3,136)=2.63$, $\left.p=0.053, R^{2}=0.055\right]$. Adding gender, age and BPD severity in block 2 revealed also no significant model $[F(3,136)=1.71, p=0.124$, $\left.R^{2}=0.071\right]$.

The third hierarchical regression analysis, with general AAT effects (as dependent variable and BIS, BAS and EC entered in block 1, revealed a significant model $\left[F(3,136)=3.61, p=0.015, R^{2}=0.074\right]$. The level of effortful control made a significant contribution to the model $[\beta=0.251, t=2.78, p=0.006$, BIS and BAS not (both $p>0.653$ ). The model with BIS, BAS, EC, gender, age and BPD severity in block 2 was still significant $[F(3,133)=2.37, \quad p=0.033$, $\left.R^{2}=0.097\right]$. This regression model revealed that effortful control remained significantly positive associated with general AAT effect-scores towards emotional faces with directed gaze $[\beta=0.234, t=2.45$, $p=0.015]$ after controlling for gender, age and BPD severity.

To investigate which temperament dimension had the strongest association with the EST effect-scores, two separate hierarchical linear regression analyses were performed.

The first hierarchical regression analysis, with EST positive bias effect-scores (positive minus neutral) as dependent variable and BIS, BAS and EC entered in block 1, and adding gender, age and BPD severity in block 2 did not provide significant results [resp. $F$ $(3,135)=0.09, p=0.965, R^{2}=0.002 ; F(3,135)=0.29, p=0.939$, $\left.R^{2}=0.013\right]$.

The second hierarchical regression analysis, with EST negative bias effect-scores (negative minus neutral) as dependent variable and BIS, BAS and EC entered in block 1, and adding gender, age and BPD severity in block 2 did not provide significant results [resp. $F(3$, $135)=0.30, \quad p=0.823, \quad R^{2}=0.007 ; \quad F(3,135)=0.90, \quad p=0.497$, $\left.R^{2}=0.039\right]$.

\subsection{BPD subtypes and their performance on the AAT and EST}

The mean (and SE) AA effect-scores per condition of the four BPD subtypes are presented in Fig. 1. The means (and SE) of the time to name the colour of neutral, negative and positive words on the EST for the four BPD subtypes are presented in Fig. 2.

To compare the four BPD subtypes on the two experimental tasks, three hierarchical linear regression analyses were performed.

The first hierarchical regression analysis, with general AAT effectscores as dependent variable and BPD subtypes entered in block 1 as independent variable, and adding gender, age and BPD severity in block 2 , did not provide significant results [resp. $F(3,136)=1.87, p=0.138$, $\left.R^{2}=0.040 ; F(3,136)=1.79, p=0.104, R^{2}=0.075\right]$.
The second hierarchical regression analysis, with EST positive bias (positive minus neutral) as dependent variable and BPD subtypes entered in block 1, and adding gender, age and BPD severity in block 2, did not provide significant results [resp. $F(3,136)=0.52, p=0.667$, $\left.R^{2}=0.011 ; F(3,136)=0.48, p=0.824, R^{2}=0.021\right]$.

The third regression analysis with EST negative bias (negative minus neutral) as dependent variable and BPD subtypes entered in block 1, and adding gender, age and BPD severity in block 2, did not provide significant results [resp. $F(3,136)=1.20, p=0.313$, $\left.R^{2}=0.026 ; F(3,136)=1.27, p=0.276, R^{2}=0.055\right]$.

These findings revealed no significant differences between the four BPD subtypes on AAT effect-scores, EST positive and negative attentional bias.

\section{Discussion}

In this study, an investigation was conducted on the relationship between BPD temperament dimensions/subtypes and action tendencies (AAT) and attentional bias (EST) of emotional stimuli.

First, findings of the current study demonstrate a positive relationship between emotional action tendencies measured with the AAT and effortful control but no significant associations with BIS and BAS reactivity. The latter finding, confirms findings of previous studies which show no or low correlations between self-reported temperament dimensions and experimental tasks (Claes et al., 2012; Kobeleva et al., 2014; Voth et al., 2014; Roose et al., 2013). Kobeleva et al. (2014), for example, applied a joystick task measuring automatic approach/ avoidance tendencies in BPD patients and found no significant correlations between BIS/BAS scores and performance on this task. In contrast, contrary to previous findings, results of the current study demonstrated that the level of effortful control was positively related to approach-avoidance tendencies to faces when the gaze was directed towards the participants and not when the gaze was averted. This is not surprising, since direct gaze, compared to averted gaze, is detected more efficiently and is perceived as a signal to interact, which evokes more intense automatic activation (Roelofs et al., 2010). Interestingly, the level of effortful control seems to be associated with a general emotional action tendency to directed faces, even when controlling for gender, age and BPD severity. This finding suggests that BPD patients with a higher level of effortful control show a better preferential response towards emotional interpersonal cues compared to the BPD patients who have a lower level of effortful control, above and beyond gender, age and severity of BPD psychopathology. In contrast, BPD patients with lower levels of effortful control show diminished approach-avoidance tendencies towards emotional faces, which may be linked to a higher level of difficulties in acting flexibly in different situations and problematic social interactions (Radke et al., 2014). This interpretation is consistent with the report by Hoermann et al. (2005) who found the highest reported problems in interpersonal functioning in BPD patients with the lowest level of effortful control. In general, these findings are in line with previous findings of disadvantageous performance in BPD patients with low levels of effortful control on tasks capturing emotion processing (Posner et al., 2003; Preti et al., 2016).

Results of the current study did not demonstrate any relationship between attentional bias measured with the EST and any of the selfreported temperament dimensions. In addition there was no evidence to support the hypothesis that attentional bias could be explained by the level of effortful control. Despite the evidence that the EST card version that was used measured attentional bias effect (longer colour naming time for emotional words compared to neutral words) this card version, in contrast to a computerised EST version, might not be specific enough to assess reaction times and errors for this purpose. Hence, based on the current findings, it cannot be concluded that temperament dimensions are associated with attentional bias.

The second aim of the present study was to explore differences in emotional action tendencies and attentional bias between four previous 


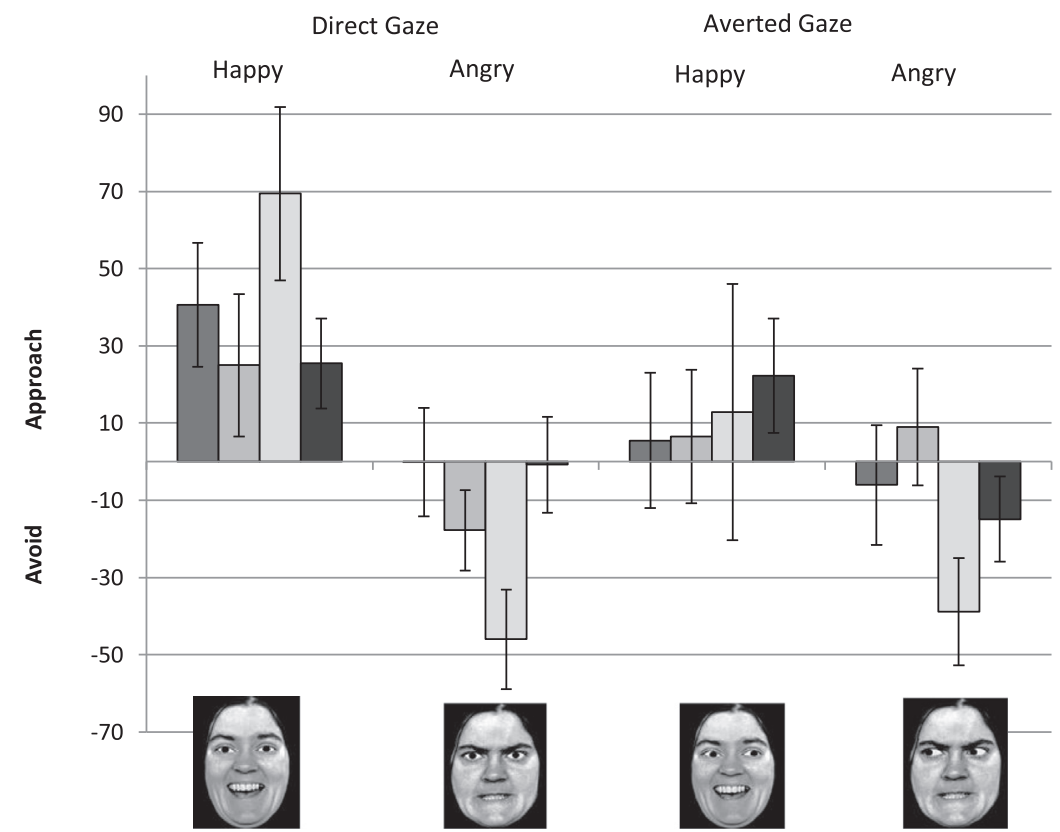

$\square$ Low Anxiety

$\square$ Inhibited

$\square$ High Self-control

Emotional/disinhibited

Fig. 1. Means (and SE) AAT effect scores (difference reaction times push and pull in ms) for direct and averted gaze stimuli for each emotion for the four BPD subtypes. Sample stimuli were obtained from Karolinska Directed Emotional Faces (Lundqvist et al., 1998) (identity AM29) with permission from the copyright holders.

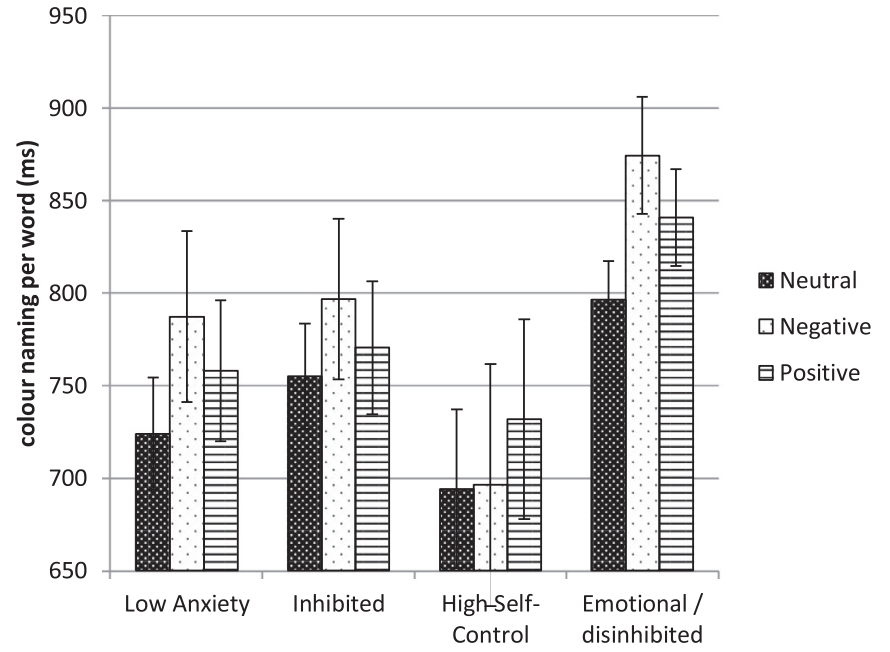

Fig. 2. Means (and SE) of the time to name the colour of neutral, negative and positive words on the EST for the four BPD subtypes.

identified BPD subtypes based on temperament dimensions. Contrary to the hypothesis, no significant differences were found between the four BPD subtypes in approach-avoidance tendencies. In addition, there were no significant differences in EST performance between the four BPD subtypes. Unfortunately, the findings of the current study could not corroborate the findings of Portella et al. (2011), who found the slowest EST performance in patients with higher severity of BPD psychopathology compared to moderate-severe BPD patients.

Despite the strengths of our study, some limitations need to be addressed. First, for the second research question, BPD subtypes with small sample sizes were included, which restrict the statistical power to detect significant differences. Second, there was an overrepresentation of female patients (almost 87\%) in the current study. Although gender was controlled in the analyses, further research should consider using a larger and more balanced sample. Despite these limitations, the data provide meaningful directions for further research. Future studies in which underlying dimensions in BPD samples are investigated, may help to further shed light into the differences between their temperament, 'severity' and responses to treatment.

To conclude, results of the current study indicate that an underlying temperament dimension of effortful control may be a critical component in emotion processing and in overall general functioning in BPD.

\section{Acknowledgements}

This study was partly supported by the Research Foundation Flanders (FWO), thanks to a Special PhD fellowship [1901317 N]. We would like to thank the three anonymous reviewers for their valuable comments on the previous version of the manuscript and Ann Morsel for increasing the readability of this manuscript.

\section{References}

Carver, C.S., White, T.L., 1994. Behavioral inhibition, behavioral activation, and affective responses to impending reward and punishment: the BIS/BAS scales. J. Pers. Soc. Psychol. 67, 319-333.

Claes, L., Mitchell, J.E., Vandereycken, W., 2012. Out of control? Inhibition processes in eating disorders from a personality and cognitive perspective. Int. J. Eating Disorders 45, 407-414.

Evans, D.E., Rothbart, M.K., 2007. Developing a model for adult temperament. J. Res. Pers. 41, 868-888.

First, M.B., Gibbon, M., Spitzer, R.L., Williams, J.B.W., Benjamin, L.S., 1997. Structured Clinical Interview for DSM-IV Axis II disorders, (SCID-II). American Psychiatric Press, Washington, DC.

Franken, I.H.A., Muris, P., Rassin, E., 2005. Psychometric properties of the Dutch BIS/BAS scales. J. Psychopathol. Behav. Assess. 27, 25-30.

Glenn, C.R., Klonsky, E.D., 2009. Emotion dysregulation as a core feature of borderline personality disorder. J. Pers. Disord. 23, 20-28.

Heuer, K., Rinck, M., Becker, E.S., 2007. Avoidance of emotional facial expressions in social anxiety: the approach avoidance task. Behav. Res. Ther. 45, 2990-3001.

Hoermann, S., Clarkin, J.F., Hull, J.W., Levy, K.N., 2005. The construct of effortful control: an approach to borderline personality disorder heterogeneity. Psychopathology 38, 82-86.

Kaiser, D., Jacob, G.A., Domes, G., Arntz, A., 2017. Attentional bias for emotional stimuli in borderline personality disorder: a meta-analysis. Psychopathology 49, 383-396.

Kobeleva, X., Seidel, E., Kohler, C., Schneider, F., Habel, U., Derntl, B., 2014. Dissociation of explicit and implicit measures of the behavioral inhibition and activation system in borderline personality disorder. Psychiatry Res 218, 134-142.

Lundqvist, D., Flykt, A., Öhman, A., 1998. The Karolinska Directed Emotional Faces (KDEF). Department of Neurosciences Karolinska Hospital, Stockholm.

Price, R.B., Siegle, G., Mohlman, J., 2012. Emotional stroop performance in older adults: effects of habitual worry. Am. J. Geriatr. Psychiatry 20, 798-805.

Portella, M.J., Soler, J., Tejero, A., Barrachina, J., Tiana, T., Pascual, J.C., et al., 2011. Slow processing in borderline personality disorder: the Emotional Stroop Paradigm. Actas Esp. Psiquiatr 39, 356-362. 
Posner, M.I., Rothbart, M.K., Vizueta, N., Thomas, K.M., Levy, K.N., Fossella, J., et al., 2003. An approach to the psychobiology of personality disorders. Dev. Psychopathol. $15,1093-1106$.

Preti, E., Richetin, J., Suttora, C., Pisani, A., 2016. Individual differences in components of impulsivity and effortful control moderate the relation between borderline personality traits and emotion recognition in a sample of university students. Psychiatry Res 238, 109-115.

Radke, S., Guths, F., Andre, J.A., Muller, B.W., de Bruijn, E.R.A., 2014. In action or in action? Social approach-avoidance tendencies in major depression. Psychiatry Res. 219, 513-517.

Radke, S., Roelofs, K., de Bruijn, E.R.A., 2013. Acting on anger: social anxiety modulates approach-avoidance tendencies after oxytocin administration. Psychol. Sc. 24, 1573-1578.

Roelofs, K., Minelli, A., Mars, R.B., van Peer, J.M., Toni, I., 2009. On the neural control of social emotional behaviour. Soc. Cogn. Affect. Neurosci. 4, 50-58.

Roelofs, K., Putman, P., Schouten, S., Lange, W.G., Volman, I., Rinck, M., 2010. Gaze direction differentially affects avoidance tendencies to happy and angry faces in socially anxious individuals. Behav. Res. Ther 48, 290-294.

Roose, A., Bijttebier, P., Van der Oord, S., Claes, L., Lilienfeld, S.O., 2013. Psychopathic traits in youth and associations with temperamental features. J. Individual Differences 34, 1-7.

Rosenthal, M.Z., Gratz, K.L., Kosson, D.S., Cheavens, J.S., Lejuez, C.W., Lynch, T.R., 2008. Borderline personality disorder and emotional responding: a review of the research literature. Clin. Psychol. Rev. 28, 75-91.

Rotteveel, M., Phaf, R.H., 2004. Automatic affective evaluation does not automatically predispose for arm flexion and extension. Emotion 4, 156-172.

Schmahl, C., Herpertz, S., Bertsch, K., Ende, G., Flor, H., Kirsch, P., et al., 2014.

Mechanisms of disturbed emotion processing and social interaction in borderline personality disorder: state of knowledge and research agenda of the German Clinical Research Unit. Borderline Pers. Disord. Emotion Dysregul. 1, 1-17.

Schotte, C.K.W., De Doncker, D., 1994. ADP-IV vragenlijst. Antwerpen: Universitair Ziekenhuis Antwerpen.

Sleuwaegen, E., Claes, L., Luyckx, K., Berens, A., Vogels, C., Sabbe, B., 2017. Subtypes in borderline patients based on reactive and regulative temperament. Pers. Individ. Differ. 108, 14-19.

Suvak, M.K., Sege, C.T., Sloan, D.M., Shea, M.T., Yen, S., Litz, B.T., 2012. Emotional processing in borderline personality disorder. Pers. Disord. 3, 273-282.

Unoka, Z., Richman, M., 2016. Neuropsychological deficits in BPD patients and the moderator effects of co-occurring mental disorders: a meta-analysis. Clin. Psychol. Rev. 44, 1-12.

Voth, E.M., Claes, L., Georgiadou, E., Selle, J., Trotzke, P., Brand, M., de Zwaan, M. Müller, A., 2014. Reactive and regulative temperament in patients with compulsive buying and non-clinical controls measured by self-report and performance-based tasks. Compr. Psychiatry 55, 1505-1512.

Williams, J.M., Mathews, A., MacLeod, C., 1996. The Emotional Stroop Task and psychopathology. Psychol. Bull. 120, 3-24.

Winter, D., 2016. Attention to emotional stimuli in borderline personality disorder - a review of the influence of dissociation, self-reference, and psychotherapeutic interventions. Borderline Pers. Disord. Emotion Dysregul. 3, 1-12. 